\title{
TBK11, a Tobacco Kinesin-14-II, Associates with the Nuclear Envelope through Its Central Coiled-Coil Domain
}

\author{
Hiroki Yasuhara* and Kazuki Kitamoto ${ }^{\dagger}$ \\ Department of Life Science and Biotechnology, Faculty of Chemistry, Materials and Bioengineering, Kansai University, \\ 3-3-35 Yamate-cho, Suita, Osaka 564-8680, Japan \\ Received May 6, 2019; accepted May 20, 2019
}

\begin{abstract}
Summary Kinesin-14-II is a plant-specific subclass of the kinesin-14 subfamily and includes kinesins with a calponin homology domain (KCHs). A tobacco member of kinesin-14-II, TBK11 is a viable candidate responsible for microtubule-dependent nuclear movement because of its apparent localization to the nuclear envelope. In tobacco BY-2 cells, it has been reported that the nuclear migration from the cell periphery to the central region in the pre-mitotic phase requires microtubules; however, the contribution of microtubules to the movement of daughter nuclei away from the cell plate in the post-cytokinetic phase has not been clarified. In the present study, we performed truncation analysis of TBK11 and found that TBK11 associates with the nuclear envelope via its central coiled-coil domain. We also found that the movement of daughter nuclei away from the cell plate is inhibited by treatment with microtubule inhibitors. These results suggest that TBK11 potentially plays a role in pre-mitotic and post-cytokinetic nuclear movement through interaction with the nuclear envelope via its central coiled-coil domain.
\end{abstract}

Key words Plant nucleus, Nuclear positioning, Microtubule, Kinesin-14-II, KCH, Tobacco BY-2 cell.

Diverse kinesin motor proteins encoded by eukaryotic genomes are classified into 14 families, i.e., kinesin-1 to kinesin-14, and some ungrouped orphans (Lawrence et al. 2001). Within these families, kinesin-14 motors show an outstanding feature; specifically, they move towards the microtubule minus end. Land plants have a large number of kinesin-14 members compared to the other organisms (Reddy and Day 2001, Richardson et al. 2006). It has been proposed that the expansion of kinesin-14s is attributed to the loss of minus end-directed motor dynein and that kinesin-14s substitute for dynein in plant cells (Vale 2003). This idea was supported by recent findings that some members of plant kinesin-14 move processively along microtubules to their minus ends in vivo and in vitro (Klotz and Nick 2012, Jonsson et al. 2015, Tian et al. 2015, Yamada et al. 2017, Tseng et al. 2018) and transport certain intracellular cargoes (Yamada et al. 2017, Yamada and Goshima 2018). Plant kinesin-14s are further divided into 6 subclasses, i.e., kinesin-14-I to kinesin-14-VI (Shen et al. 2012). One of these subclasses, kinesin-14-II, is plant specific and includes a relatively large number of members. In Arabidopsis thaliana, 9 out of 21 kinesin-14s are classified into kinesin-14-II (Shen et al. 2012). Most of the

\footnotetext{
*Corresponding author, e-mail: yasuhara@kansai-u.ac.jp

† Present address: Yao School for Special Needs Education, 7-6 Kaminoshima-cho minami, Yao, Osaka 581-0846, Japan DOI: $10.1508 /$ cytologia. 84.285
}

kinesin-14-IIs possess a calponin homology $(\mathrm{CH})$ domain in their $\mathrm{N}$-terminal region and are named $\mathrm{KCHs}$ for kinesins with a calponin homology domain (Preuss et al. 2004). In A. thaliana, 7 out of 9 kinesin-14-IIs possess a $\mathrm{CH}$ domain (Richardson et al. 2006).

Since the $\mathrm{CH}$ domain is known to be an actin-binding motif of a variety of actin-associated proteins (Gimona et al. 2002, Korenbaum and Rivero 2002), KCHs potentially act as dynamic linkers between microtubules and actin filaments. In fact, several $\mathrm{KCHs}$ were reported to interact with both microtubules and actin filaments in vivo and in vitro (Preuss et al. 2004, Frey et al. 2009, Xu et al. 2009, Buschmann et al. 2011, Umezu et al. 2011) and to transport actin filaments along microtubules in vitro (Walter et al. 2015, Tseng et al. 2018). The KCHs of flowering plants have been implicated in various cellular events, including cell elongation (GhKCH1; Preuss et al. 2004, GhKCH2; Xu et al. 2009, OsKCH1; Frey et al. 2010), pre-mitotic nuclear migration (OsKCH1; Frey et al., 2010), mitochondrial respiration (A. thaliana KP1; Yang et al. 2011) and intra- and intercellular transport of a transcription factor (A. thaliana KinG; Spiegelman et al. 2018). Recently, KCHs of the moss Physcomitrella patens have been reported to be involved in nuclear transport and apical cell tip growth (Yamada and Goshima 2018).

We reported in a preceding paper (Yasuhara and Kurisu 2019) that tobacco BY-2 kinesin like-polypeptide 11 (TBK11) clearly localized to the nuclear envelope throughout the cell cycle in BY-2 cells. Although TBK11 


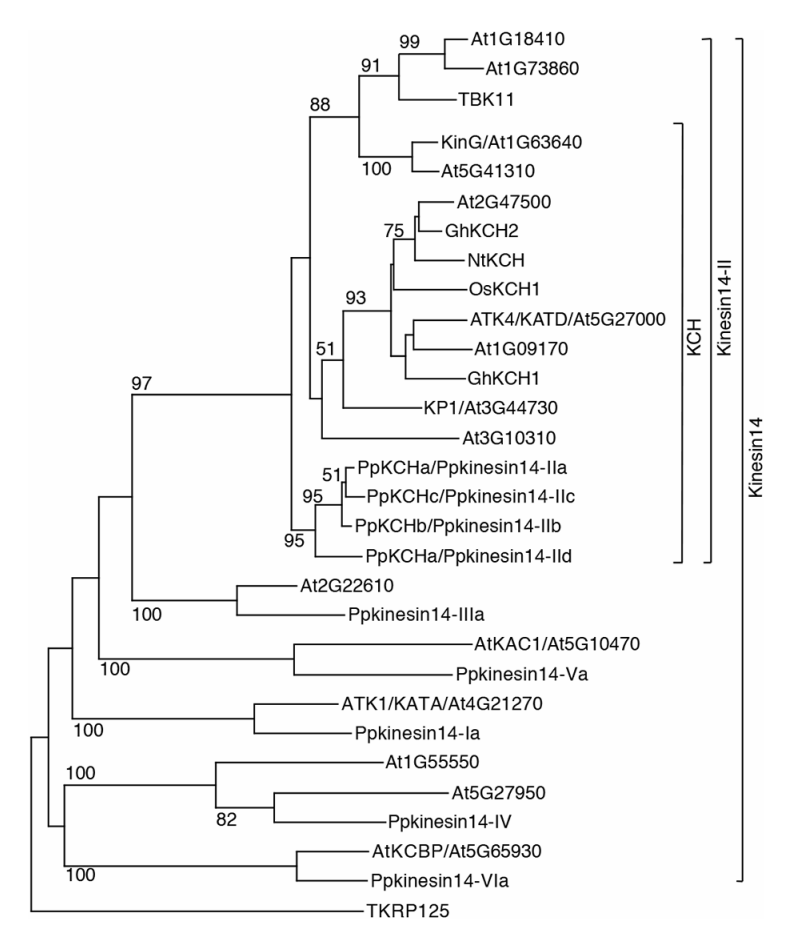

Fig. 1. Phylogenetic relationships of TBK11 and representatives of plant kinesin-14s. The AGI numbers of Arabidopsis thaliana (At) kinesins are given in the tree. The Phypa numbers of kinesins from Physcomitrella patens (Pp) and the GenBank accession numbers of kinesins from Oryza sativa (Os), Gossypium hirsutum (Gh) and Nicotiana tabacum (Nt) are as follows: Ppkinesin14-Ia, Pp3c7_11530; PpKCHa/ Ppkinesin14-IIa, Pp3c14_19550; PpKCHb/Ppkinesin14IIb, Pp3c2_9150; PpKCHc/Ppkinesin14-IIc, Pp3c17_21780; PpKCHd/Ppkinesin14-IId, Pp3c24_19380; Ppkinesin14IIIa, Pp3c24_13720; Ppkinesin14-IV, Pp3c10_8460; Ppkinesin14-Va, Pp3c3_10920; Ppkinesin14-VIa, Pp3c15_3730; OsKCH1, AK065586; GhKCH1, AY695833; GhKCH2, EF432568; TBK11, LC482136; NtKCH, JF835913; TKRP125, D83711. Only bootstrap values greater than 50 were shown on the tree. Horizontal branch length is proportional to the estimated evolutionary distance.

possesses a $\mathrm{CH}$ domain in its N-terminal region, phylogenetic analysis showed that TBK11 forms a clade with the two $A$. thaliana kinesin-14-IIs that lack a $\mathrm{CH}$ domain (Fig. 1). To our knowledge, none of the kinesin-14-IIs belonging to this clade have been studied in flowering plants. In the present study, we performed truncation analysis of TBK11 results for the nuclear envelopebinding domain of TBK11. Furthermore, to determine whether microtubule motor kinesins including TBK11, potentially play a role in the nuclear migration after cytokinesis, we examined the effects of microtubule inhibitors on the movement of daughter nuclei away from the cell plate.

Materials and methods

\section{Plant materials}

Tobacco BY-2 cells (Nicotiana tabacum L. cv. Bright Yellow 2) were cultured at $27^{\circ} \mathrm{C}$ in suspension using modified Linsmaire and Skoog's medium, as described by Nagata et al. (1981).

\section{Phylogenetic analysis}

Amino acid sequences of kinesin motor domains were aligned using MAFFT ver. 6.864 (https://www.genome. $\mathrm{jp} /$ tools-bin/mafft). A phylogenetic analysis was performed with SEQBOOT, PROML and CONSENSE programs contained in the PHYLIP ver. 3.68 package and the tree was drawn by the NJ-prot program. It was rooted arbitrarily using TKRP125 as an outgroup. Statistical support for internal branches by bootstrap analyses was calculated using 100 replications with the SEQBOOT, PROML and CONSENSE programs.

\section{Construction of plasmids}

pMDC7851Y: The YFP sequence was amplified from the p35SYFPN21 vector (Yasuhara and Oe 2011) using the following primer pair: AscI-linker-YFP_F and SpeTAA-YFP_R. The PCR fragment of YFP was digested with AscI and SpeI and ligated into the pMDC7851 vector (Yasuhara and Kurisu 2019), replacing the AscI-SpeI fragment of GFP6His to generate a pMDC7851Y vector.

Binary plasmids for inducible expression of Cterminal fluorescent protein-tagged mutant TBK11: The DNA fragments encoding TBK11CH, TBK11CH$\mathrm{CC}$, and TBK11CC were amplified using the following primer pairs: attB1-TBK11FL-F and attB2-TBK11CHR; attB1-TBK11FL-F and attB2-TBK11CHCC-R; attB1TBK11CC-F and attB2-TBK11CH-CC-R, respectively. These attB site-attached PCR fragments were cloned into the $\mathrm{pDONR} / \mathrm{Zeo}$ entry vector (Invitrogen) using BP clonase (Invitrogen) to generate plasmids, pDONRTBK11CH, pDONR-TBK11CH-CC, and pDONR-TBK11CC, respectively.

DNA fragments encoding the mutant TBK11 with truncation other than described above was prepared by recombinant PCR as described below. The DNA fragment encoding TBK11 $\triangle \mathrm{CH}$ was amplified using pDONR-F and pDONR-R from a mixture of two PCR fragments amplified from pDONR-TBK11FL using the two primer pairs: pDONR-F and TBK11 $\triangle \mathrm{CH}-\mathrm{R}$; and TBK11 $\triangle \mathrm{CH}-\mathrm{F}$ and pDONR-R. The DNA fragments encoding TBK11 $\Delta \mathrm{MD}$, TBK11 $\mathrm{CCC}$ and TBK11T620N were amplified in the same way using the primers: TBK11 $\triangle \mathrm{MD}-\mathrm{R}$ and TBK11 $1 \mathrm{MD}-\mathrm{F}$; TBK11 $\Delta \mathrm{CC}-\mathrm{R}$ and TBK11 $\triangle \mathrm{CC}-\mathrm{F}$; and TBK11T620N-R and TBK11T620N-F, respectively in place of TBK11 $\Delta \mathrm{CH}-\mathrm{R}$ and TBK11 $\Delta \mathrm{CH}-\mathrm{F}$. The DNA fragments encoding TBK11 mutants were further cloned into the pMDC7851 or pMDC7851Y vector using LR clonase to generate plasmids pMDC7851-TBK11FL, pMDC7851-TBK11 $\Delta \mathrm{CH}$, pMDC7851-TBK11 $\Delta \mathrm{MD}, \quad$ pMDC7851-TBK11 $\Delta \mathrm{CC}$, pMDC7851Y-TBK11CH-CC, pMDC7851Y-TBK11CH, pMDC7851Y-TBK11CC and pMDC7851-TBK11T620N.

pMDC100-YFPTUB-H2BtdT: The 35Sp-histoneH2B-tdTomato-NOSt region of the pENTRHistoneH2B- 
Table 1. The sequences of PCR primers used in this study.

\begin{tabular}{|c|c|}
\hline Primer name & Sequence \\
\hline AscI-linker-YFP_F & 5'-gcagGGCGCGCCggtgctggagetggGATGGTGAGCAAGGGCGAGGAGCTGT-3' \\
\hline Spe-TAA-YFP_R & 5'-gcagACTAGTTTACTTGTACAGCTCGTCCATGCCGAGAGTG-3' \\
\hline attB1-TBK11FL-F & 5'-GGGGACAAGTTTGTACAAAAAAGCAGGCTAACAATGAATTCAATACTGGATCGCGTAGCTAAAG-3' \\
\hline attB2-TBK11CH-R & 5'-GGGGACCACTTTGTACAAGAAAGCTGGGTCCTACAGGACTTCTCAAAGCCCGCTGGA-3' \\
\hline attB2-TBK11CHCC-R & 5'-GGGGACCACTTTGTACAAGAAAGCTGGGTCCGCGATTCTCAGCAAGGACGGCAGAG-3' \\
\hline attB1-TBK11CC-F & 5'-GGGGACAAGTTTGTACAAAAAAGCAGGCTAACAATGGCAGAGCCATCAGCTGCATTGTTA-3' \\
\hline pDONR-F & 5'-CGCTAGCATGGATGTTTTCCCAGTCAC-3' \\
\hline pDONR-R & 5'-GAGATTTTGAGACACGGGCCAGAGC-3' \\
\hline TBK11 $\Delta \mathrm{CH}-\mathrm{R}$ & 5'-CСATCTCСТCCAACATTCGGCATCTTAGCATTACTACTTGACAAAGGCTCAAAGATG-3' \\
\hline TBK11 $\triangle \mathrm{CH}-\mathrm{F}$ & 5'-ATGCCGAATGTTGGAGGAGATGG-3' \\
\hline TBK11 $1 \mathrm{MD}-\mathrm{R}$ & 5'-GCCGTCTTTGCTACTTCGAGCAGCATTTCCTTTTAACTCCTGGACCTCATTGTGTAGTTT-3' \\
\hline TBK11 $\Delta \mathrm{MD}-\mathrm{F}$ & 5'-GCTGCTCGAAGTAGCAAAGACGGC-3' \\
\hline TBK11 $\Delta \mathrm{CC}-\mathrm{R}$ & 5'-AGAGATTCGCCGCTCAATTTCTTGC-3' \\
\hline TBK11 $1 \mathrm{CC}-\mathrm{F}$ & 5'-GCAAGAAATTGAGCGGCGAATCTCTTCACAGAGGTGGAGCAAGAAGGAGAA-3' \\
\hline TBK11T620N-R & 5'-CAGGGCCAGTCATGGTATAGTTTTTCCCTG-3' \\
\hline TBK11T620N-F & 5'-CAGGGAAAAACTATACCATGACTGGCCCTG-3' \\
\hline
\end{tabular}

tdTomato (Hayashi et al. 2007) was cloned into the Gateway cloning sites of the pMDC100-YFPTUB (Yasuhara and Kitamoto 2014) using LR clonase (Invitrogen) to generate a pMDC100-YFPTUB-H2BtdT plasmid.

The integrity of all constructs was verified by sequencing. The sequences of the PCR primers used in this study are shown in Table 1.

\section{Generation and observation of transformed BY-2 cells}

Transgenic BY-2 cells were generated by Agrobacteri$u m$-mediated transformation using the LBA4404 strain. BY-YTHR1 cells stably expressing YFP- $\beta$-tubulin and HistoneH2B-tdTomato were generated by transforming BY-2 cells with the pMDC100-YFPTUB-H2BtdT plasmid. Cells for inducible expression of fluorescent protein-tagged mutant TBK11 were generated by transforming BY-2 cells or BY-CT1 cells (Yasuhara and Kurisu 2019) with the plasmid: pMDC7851-TBK11 $\Delta \mathrm{CH}$, pMDC7851-TBK11 $\mathrm{MD}$, pMDC7851-TBK11 $\mathrm{CC}$, pMDC7851Y-TBK11CH-CC, pMDC7851Y-TBK11CH, pMDC7851Y-TBK11CC or pMDC7851-TBK11T620N. Expression of the fluorescent protein-tagged TBK11 mutants was induced with $5 \mu \mathrm{M} \beta$-estradiol (Wako Pure Chemical Industries, Ltd.). Transformed BY-2 cells were observed as described previously (Yasuhara and Kurisu 2019).

Results and discussion

Subcellular localization of fluorescent protein-tagged truncated TBK11 proteins

To examine the domains required for nuclear envelope localization, plasmids for the inducible expression of C-terminal fluorescent protein-tagged truncated TBK11 proteins were constructed and transformed into BY-CT1 cells. Fluorescent protein-tagged truncated TBK11 proteins, TBK11 $\Delta$ CH-GFP, TBK11 $\Delta$ MD-GFP and TBK11CH-CC-YFP localize to the nuclear envelope (Fig. 2), indicating that the $\mathrm{CH}$ domain, the motor domain and the $\mathrm{C}$-terminal short coiled-coil domain were not required for nuclear envelope localization. On the other hand, TBK11 $\triangle \mathrm{CC}$-GFP did not localize to the nuclear envelope and dispersed throughout the cytoplasm (Fig. 2), indicating the requirement of the central coiledcoil domain for nuclear envelope localization. Furthermore, YFP-tagged TBK11CC, a small truncated TBK11 polypeptide lacking both $\mathrm{N}$-terminal and $\mathrm{C}$-terminal domains but containing the central coiled-coil domain, localized to the nuclear envelope (Fig. 2). These results suggest that the central coiled-coil domain of TBK11 is responsible for the nuclear envelope localization. We are planning to examine whether the central coiled-coil domain of TBK11 interacts with known outer membrane proteins and to search for TBK11 interacting proteins using its central coiled-coil domain as a probe.

TBK11 possesses a $\mathrm{CH}$ domain in its $\mathrm{N}$-terminal region; however, neither TBK11FL-GFP nor GFP-TBK11FL showed a filamentous localization pattern (Yasuhara and Kurisu 2019), indicating that these GFP-tagged TBK11 proteins did not associate with actin filaments. In the present study, none of the fluorescent protein-tagged truncated TBK11 polypeptides, including TBK11CH, a small N-terminal TBK11 fragment including the $\mathrm{CH}$ domain, showed an interaction with actin filaments (Fig. 2). Although the sequence homology analysis detected a $\mathrm{CH}$ domain in TBK11, its actin-binding property does not seem to be conserved.

It has been reported that the overexpression of GFPtagged OsKCH, a rice counterpart of TBK $9 / \mathrm{NtKCH}$ (Klotz and Nick 2012), affects pre-mitotic nuclear positioning in BY-2 cells (Frey et al. 2010). Phylogenetic analysis of the motor domain sequence (Fig. 1) has indicated that TBK11 is divergent from TBK9/NtKCH. The $\mathrm{CH}$ domain of TBK11 did not interact with actin filaments (Fig. 2). Furthermore, TBK9/NtKCH did not have a central coiled coil domain for interaction with the nuclear envelope. Therefore, if TBK11 plays a role in pre-mitotic nuclear positioning, these facts suggest that TBK11 and TBK9/NtKCH do not share common mecha- 


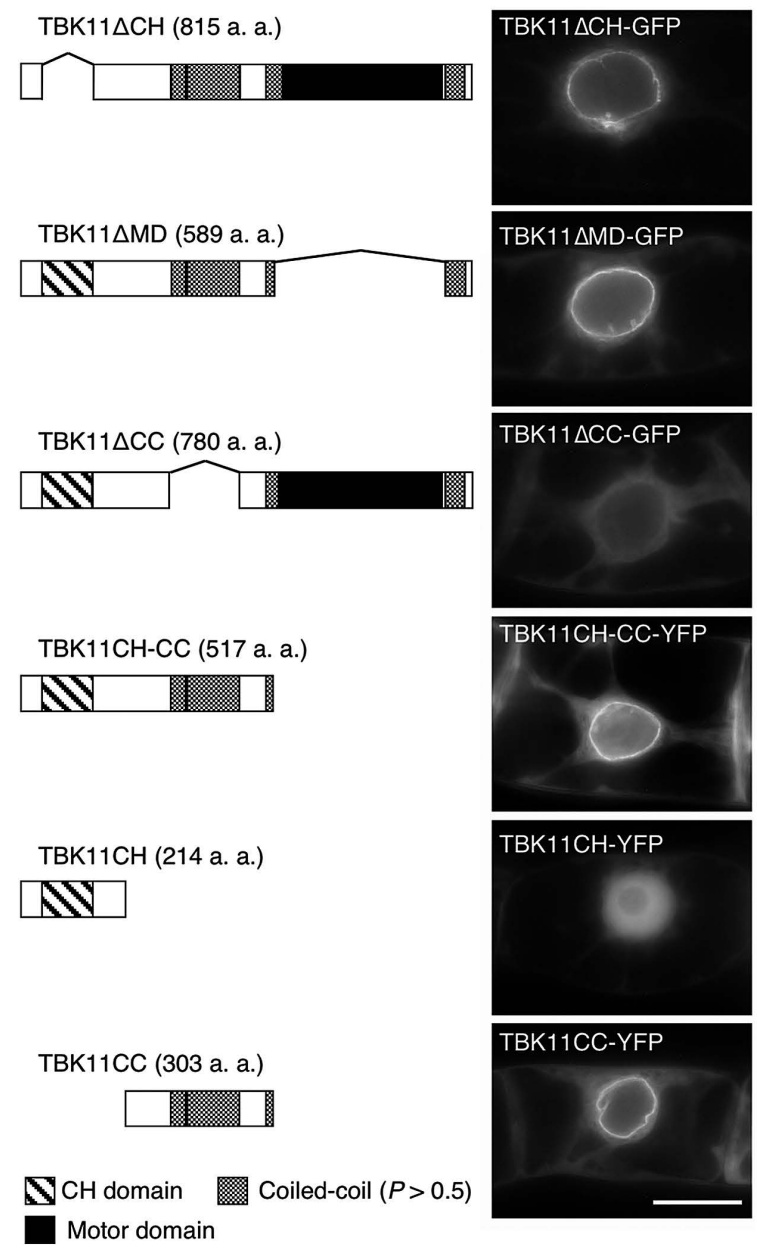

Fig. 2. Localization of GFP/YFP-tagged truncated-TBK11 mutants. Left: Schematics of TBK11 mutants. Calponin homology domains ( $\mathrm{CH}$ domain, hatched box), coiled-coils (Probability $>0.5$, stippled boxes), and kinesin motor domains (solid box) are indicated. Right: Localization of the C-terminal GFP/YFP fusion proteins of TBK11 mutants. The lack of the $\mathrm{CH}$ domain $(\mathrm{TBK} 11 \Delta \mathrm{CH})$ or the motor domain (TBK11 $\triangle \mathrm{MD}$ ) did not affect the localization of TBK11 to the nuclear envelope. The lack of the central coiled-coil domains, located on the N-terminal side of the motor domain (TBK11 $\triangle \mathrm{CC}$ ), resulted in loss of the localization of TBK11 to the nuclear envelope. The N-terminal region outside of the motor domain (TBK11CH-CC) and the coiled-coil domains, located on the $\mathrm{N}$-terminal side of the motor domain (TBK11CC), localized to the nuclear envelope, but the $\mathrm{CH}$ domain alone $(\mathrm{TBK} 11 \mathrm{CH})$ did not. Scale bar $=20 \mu \mathrm{m}$.

nisms for nuclear positioning.

GFP-tagged TBK11 mutant TBK11T620N-GFP was localized to the nuclear envelope as well as along microtubules

The $\mathrm{T}$ to $\mathrm{N}$ amino acid substitution at the ATP binding motif of the kinesin motor domain is a rigor-type mutation in which kinesins bind tightly to microtubules and do not move along microtubules (Nakata and Hirokawa 1995). Because the ATP binding motif is conserved in TBK11, we examined the localization of C-terminal GFP-tagged TBK11 with the T620N mutation (TBK11T620N-GFP) to determine whether the motor domain of TBK11 has the ability to interact with microtubules. A plasmid for the inducible expression of TBK11T620N-GFP was constructed and transformed into BY-CT1 cells and generated CT-TBK11T620N-GFP cells. Similar to the fluorescent-protein tagged wild-type TBK11 (Yasuhara and Kurisu 2019), TBK11T620NGFP localized to the nuclear envelope (Fig. 4, a1, a'1, c1). Dual observation of mCherry- $\beta$-tubulin and TBK11T620N-GFP revealed that TBK11T620N-GFP also localized along cortical microtubules in interphase cells, spindle microtubules in mitotic cells and phragmoplast microtubules in cytokinetic cells (Fig. 4). These observations indicated that the $\mathrm{T} 620 \mathrm{~N}$ mutation promoted the microtubule-binding activity of TBK11, and thus TBK11T620N displayed an ability to interact with microtubules.

Nuclear migration after cytokinesis requires microtubule integrity

During the centrifugal development of phragmoplasts in the cytokinesis of BY-2 cells, when the microtubules in the central region of the phragmoplast disassemble, the daughter chromosomes/nuclei move close to the cell plate, and then, after the completion of cell-plate formation, the daughter nuclei move away from the cell plate. Because TBK11 was localized to the nuclear envelope throughout the cell cycle (Yasuhara and Kurisu 2019), TBK11 could be a candidate responsible for this nuclear migration event. Nuclear migration from the cell periphery to the central region in the pre-mitotic phase requires both microfilaments and microtubules in BY-2 cells (Katsuta et al. 1990, Miyake et al. 1997). However, the post-cytokinetic nuclear migration event was reported to depend on actin filaments in BY-2 cells (Higaki et al. 2006), and the contribution of microtubules to this event has not been clarified. Therefore, we examined the effects of microtubule inhibitors on nuclear migration after cytokinesis using BY-YTHR1 cells, in which chromosomes and microtubules were visualized with histone H2B-tdTomato and YFP- $\beta$-tubulin (Fig. 3). Because microtubule inhibitors inhibit mitosis and cytokinesis, treatment with the drugs was initiated immediately after the completion of cytokinesis. In the control experiments, the nuclei moved away from the cell plates at a velocity of approximately $0.3 \mu \mathrm{m} / \mathrm{s}$ (Fig. 3), which was equivalent to that reported previously (Higaki et al. 2006). Treatment of cells with $20 \mu \mathrm{M}$ propyzamide inhibited this nuclear movement up to approximately 30 min after the fusion of the cell plate with the parental cell wall, and furthermore, treatment with $20 \mu \mathrm{M}$ oryzalin completely inhibited this nuclear movement during the observation period (Fig. 3). These observations indicate the requirement of microtubule integrity for nuclear movement away from the cell plate. Therefore, TBK11 potentially plays a role in the pre-mitotic and post-cytokinetic nuclear migration events. 
A

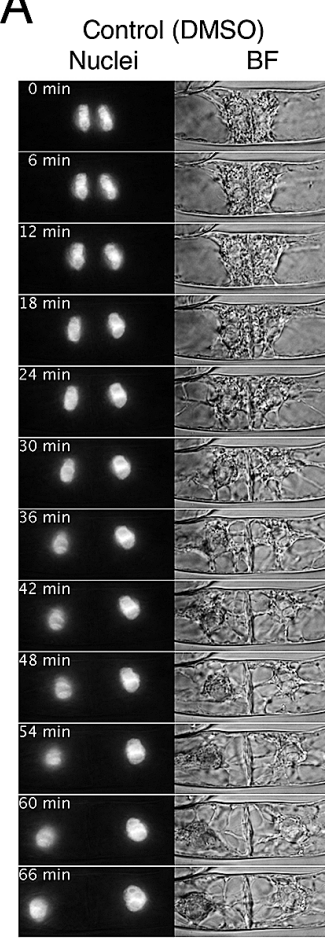

$20 \mu \mathrm{M}$ propyzamide

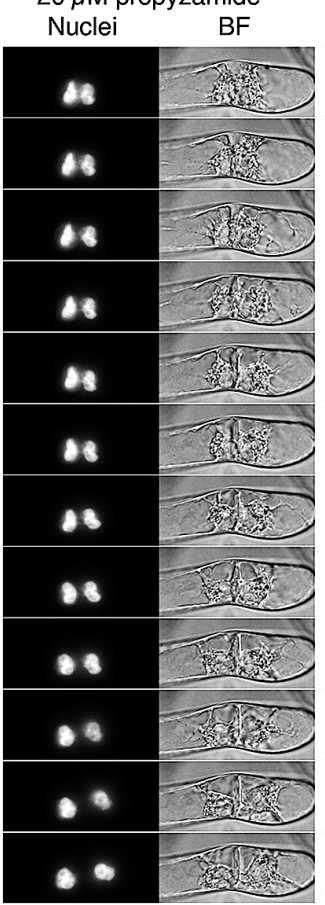

$20 \mu \mathrm{M}$ oryzalin Nuclei BF

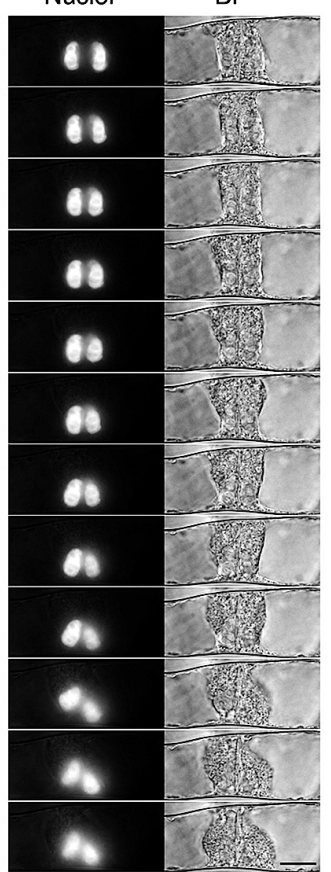

B

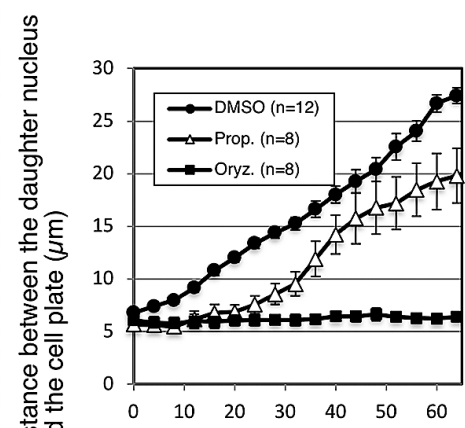

Time after the fusion of the cell plate with the parental cell wall (min)

Fig. 3. Effects of microtubule inhibitors on nuclear migration after cytokinesis. Cells were observed on an inverted fluorescence microscope, and images were recorded at 2 min intervals. (A) Time-sequential images of nuclei (A, Nuclei). Corresponding bright-field images (BF) are also shown. Time after the fusion of the cell plate with the parental wall is indicated on the left. BY-YTHR1 cells constitutively expressing YFP- $\beta$-tubulin and histone H2B-tdTomato were used. During the course of the observation, when the growing margins of the cell plate reached the parental cell wall, the medium was replaced with a medium containing $20 \mu \mathrm{M}$ propyzamide or $20 \mu \mathrm{M}$ oryzalin. In the control experiment, the medium was replaced with a medium containing a corresponding amount of DMSO. (B) Changes in the distance between the daughter nucleus and the cell plate after cytokinesis in control (DMSO), propyzamide- (Prop.) and oryzalin- (Oryz.) treated BY-TYHR1 cells. The mean \pm SE was plotted against time after the fusion of the cell plate with the parental wall. Both propyzamide and oryzalin inhibited nuclear migration away from the cell plate.

\section{Determination of the role of TBK11 in nuclear migration}

At the beginning of our study, we expected that the overexpression of truncated TBK11 fragments that lack the motor domain but have a nuclear envelope binding domain might block the binding sites on the nuclear surface for endogenous TBK11 and thus might affect nuclear positioning. However, the expression of fluorescent protein-fused truncated TBK11 mutants, including TBK11CC, a short fragment containing a nuclear binding domain, did not cause conspicuously abnormal nuclear positioning. Although we cannot rule out the possibility that the expression level of truncated TBK11 fragments was not enough to block the putative binding sites for endogenous TBK11, these results suggest the presence of functionally redundant proteins that do not compete for binding sites on the nuclear surface with TBK11. kinesin-ARKs (Miki et al. 2015) and KCBPs (Yamada et al. 2017) have been reported to have a role in nuclear migration after cytokinesis in protonema cells of $P$. patens. Since these kinesins do not share a homology with TBK11 outside the motor domain and KCBPs have been suggested to interact directly with the nuclear membrane (Yamada et al. 2017), the tobacco counterparts of these kinesins would not compete for binding sites on the nuclear surface with TBK11. Therefore, these kinesins seem to be candidates for proteins which have a redundant role in nuclear migration after cytokinesis with TBK11 in tobacco BY-2 cells. In protonema cells of $P$. patens, daughter nuclei start to move before completion of cytokinesis and were associated with longitudinal cytoplasmic microtubules during their migration (Miki et al. 2015, Yamada et al. 2017). On the other hand, in tobacco BY-2 cells, daughter nuclei start to move after the completion of cell-plate formation in parallel to the formation of radial microtubule arrays emerging from the daughter nuclei surface, and the association of nuclei with longitudinal cytoplasmic microtubules has not been observed during daughter nuclear migration (Kumagai et al. 2001, Yoneda and Hasezawa 2003). These differences support the idea that the mechanisms for daughter nuclei migration are not conserved between protonema cells of $P$. patens and tobacco BY-2 cells; thus, different types of kinesins have a role in these events.

Recently, Yamada and Goshima (2018) reported that a knockout of all four KCHs of P. patens (PpKCHs) results in aberrant nuclear positioning. They showed that the nucleus was positioned near the apical cell wall in the protonemal apical cells of the quadruple KO line, while 


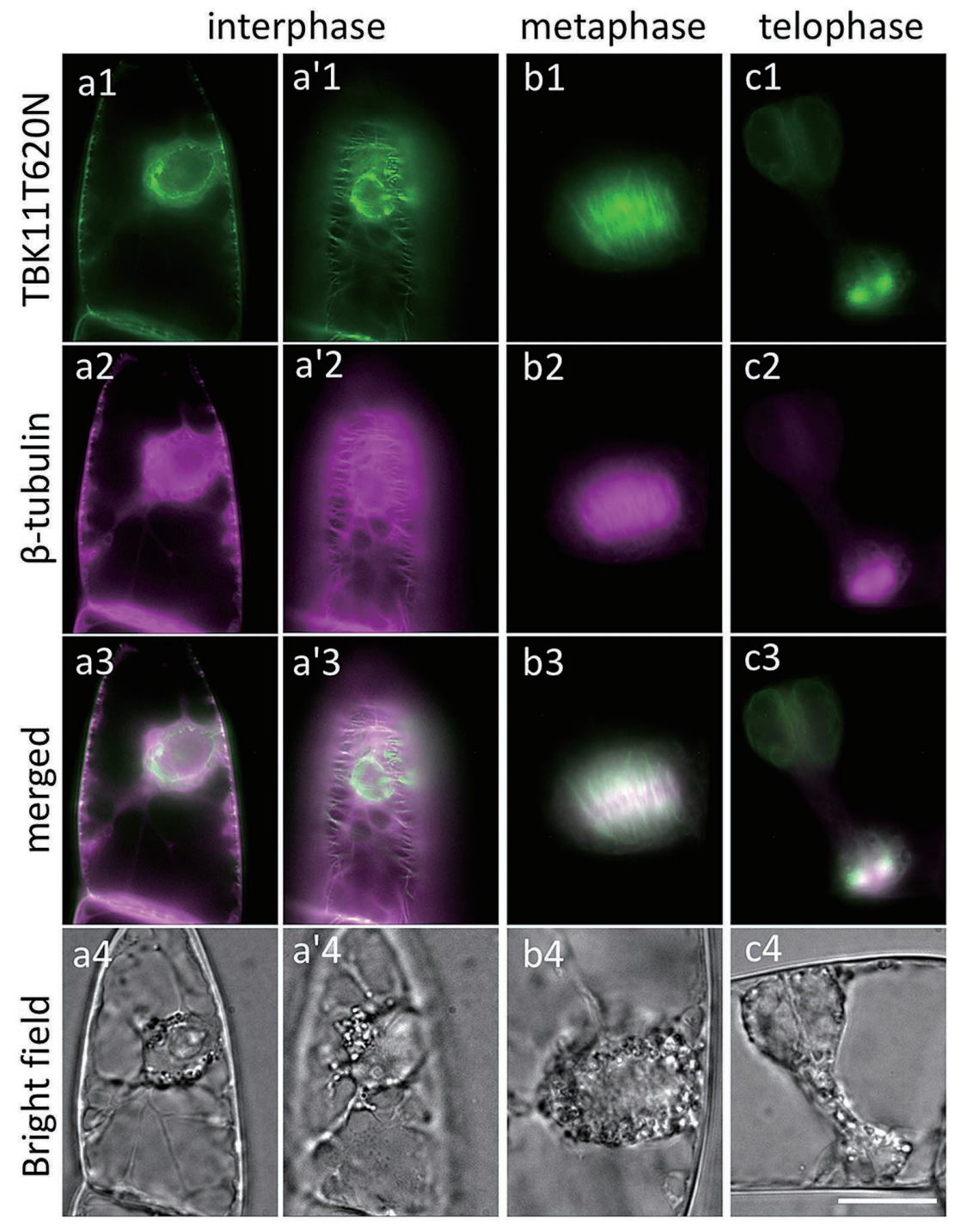

Fig. 4. Localization of TBK11T620NGFP during the cell cycle in BY-2 cells. TBK11T620N is a putative non-motile, rigor-type mutant that has a point mutation (T620N) within an ATP-binding consensus motif located in the motor domain. a1-c1: Images of TBK11T620N-GFP. a2-c2: Images of mCherry- $\beta$-tubulin. a3-c3: Merged images. a4-c4: Bright field images. a1-a4, a'1-a' 4 : A cell at interphase. a' $1-a^{\prime} 4$ are the surface views of the same cell in a1-a4. b1-b4: A cell at metaphase. c1-c4: A cell at telophase. TBK11T620N-GFP localized to the nuclear envelope as well as to the microtubules. Scale bar $=20 \mu \mathrm{m}$. the nucleus of control cells maintained cell centre positioning and thus suggested that the minus end-directed $\mathrm{KCHs}$ and the plus end-directed kinesin-ARKs have an antagonistic role in maintaining the cell centre positioning of the nucleus in normal protonemal cells (Yamada and Goshima 2018). Although microtubule focus formation at the protonemal apical cell tip requires both actin filaments and $\mathrm{KCHs}$, the rescue experiment of the $\mathrm{KCH}$ $\mathrm{KO}$ line by truncated $\mathrm{KCHs}$ revealed that the $\mathrm{CH}$ domain of $\mathrm{PpKCH}$ was not required for nuclear positioning (Yamada and Goshima 2018). This finding is consistent with the result that the $\mathrm{CH}$ domain of TBK11 did not interact with actin filaments (Fig. 2). In contrast to TBK11, which was clearly localized to the nuclear envelope, the enrichment of PpKCHs on the nuclear surface was not observed (Yamada and Goshima 2018). During the course of evolution from the common ancestor of flowering plants and mosses to flowering plants that involves the duplication and differentiation of $\mathrm{KCH}$ genes, TBK11 might have lost actin binding activity of the $\mathrm{CH}$ domain and might have acquired tight binding activity to the nuclear envelope to devote itself to nuclear migration.

Before we reached any conclusion on the molecular evolution of $\mathrm{KCH}$ genes and the mechanisms for microtubule-dependent nuclear positioning in BY-2 cells, we must determine whether TBK11 truly plays a role in nuclear migration. To achieve this, knockout mutants of TBK11 and potential functional homologs are required. We are now attempting to obtain these mutants by CRISPR/Cas9-mediated targeted mutagenesis in BY-2 cells.

\section{Acknowledgements}

We thank Dr. Seiichiro Hasezawa (University of Tokyo, Japan) for providing pENTR-HistoneH2B-tdTomato, Reina Kawamoto (Kansai University, Japan), Marina Sakakimoto (Kansai University), Rei Miyamoto (Kansai University), and Sho Mitsutake (Kansai University) for construction of plasmids and generation of transformed BY-2 cell lines and Dr. Tetsuhiro Asada (Osaka University, Japan) for useful discussions. 


\section{References}

Buschmann, H., Green, P., Sambade, A., Doonan, J. H. and Lloyd, C. W. 2011. Cytoskeletal dynamics in interphase, mitosis and cytokinesis analysed through Agrobacterium-mediated transient transformation of tobacco BY-2 cells. New Phytol. 190: 258-267.

Frey, N., Klotz, J. and Nick, P. 2009. Dynamic bridges-A calponindomain kinesin from rice links actin filaments and microtubules in both cycling and non-cycling cells. Plant Cell Physiol. 50: 1493-1506.

Frey, N., Klotz, J. and Nick, P. 2010. A kinesin with calponin-homology domain is involved in premitotic nuclear migration. J. Exp. Bot. 61: 3423-3437.

Gimona, M., Djinovic-Carugo, K., Kranewitter, W. J. and Winder, S. J. 2002. Functional plasticity of CH domains. FEBS Lett. 513: 98-106.

Hayashi, T., Sano, T., Kutsuna, N., Kumagai-Sano, F. and Hasezawa, S. 2007. Contribution of anaphase B to chromosome separation in higher plant cells estimated by image processing. Plant Cell Physiol. 48: 1509-1513.

Higaki, T., Kutsuna, N., Okubo, E., Sano, T. and Hasezawa, S. 2006. Actin microfilaments regulate vacuolar structures and dynamics: Dual observation of actin microfilaments and vacuolar membrane in living tobacco BY-2 Cells. Plant Cell Physiol. 47: 839-852.

Jonsson, E., Yamada, M., Vale, R. D. and Goshima, G. 2015. Clustering of a kinesin-14 motor enables processive retrograde microtubule-based transport in plants. Nat. Plants 1: 15087.

Katsuta, J., Hashiguchi, Y. and Shibaoka, H. 1990. The role of the cytoskeleton in positioning of the nucleus in premitotic tobacco BY-2 cells. J. Cell Sci. 95: 413-422.

Klotz, J. and Nick, P. 2012. A novel actin-microtubule cross-linking kinesin, $\mathrm{NtKCH}$, functions in cell expansion and division. New Phytol. 193: 576-589.

Korenbaum, E. and Rivero, F. 2002. Calponin homology domains at a glance. J. Cell Sci. 115: 3543-3545.

Kumagai, F., Yoneda, A., Tomida, T., Sano, T., Nagata, T. and Hasezawa, S. 2001. Fate of nascent microtubules organized at the M/ $\mathrm{G}_{1}$ interface, as visualized by synchronized tobacco BY-2 cells stably expressing GFP-tubulin: Time-sequence observations of the reorganization of cortical microtubules in living plant cells. Plant Cell Physiol. 42: 723-732.

Lawrence, C. J., Morris, N. R., Meagher, R. B. and Dawe, R. K. 2001. Dyneins have run their course in plant lineage. Traffic 2: 362-363.

Miki, T., Nishina, M. and Goshima, G. 2015. RNAi screening identifies the armadillo repeat-containing kinesins responsible for microtubule-dependent nuclear positioning in Physcomitrella patens. Plant Cell Physiol. 56: 737-749.

Miyake, T., Hasezawa, S. and Nagata, T. 1997. Role of cytoskeletal components in migration of nuclei during the cell cycle transition from $\mathrm{G}_{1}$ phase to $\mathrm{S}$ phase of tobacco BY-2 cells. J. Plant Physiol. 150: 528-536.

Nagata, T., Okada, K., Takebe, I. and Mitsui, C. 1981. Delivery of tobacco mosaic virus RNA into plant protoplasts mediated by reverse-phase evaporation vesicles (liposomes). Mol. Gen. Genet. 184: $161-165$.

Nakata, T. and Hirokawa, N. 1995. Point mutation of adenosine triphosphate-binding motif generated rigor kinesin that selectively blocks anterograde lysosome membrane transport. J. Cell Biol.
131: $1039-1053$

Preuss, M. L., Kovar, D. R., Lee, Y.-R. J., Staiger, C. J., Delmer, D. P. and Liu, B. 2004. A plant-specific kinesin binds to actin microfilaments and interacts with cortical microtubules in cotton fibers. Plant Physiol. 136: 3945-3955.

Reddy, A. S. and Day, I. S. 2001. Kinesins in the Arabidopsis genome: A comparative analysis among eukaryotes. BMC Genomics 2: 2.

Richardson, D. N., Simmons, M. P. and Reddy, A. S. 2006. Comprehensive comparative analysis of kinesins in photosynthetic eukaryotes. BMC Genomics 7: 18.

Shen, Z., Collatos, A. R., Bibeau, J. P., Furt, F. and Vidali, L. 2012. Phylogenetic analysis of the Kinesin superfamily from Physcomitrella. Front. Plant Sci. 3: 230.

Spiegelman, Z., Lee, C. M. and Gallagher, K. L. 2018. KinG is a plant-specific kinesin that regulates both intra- and intercellular movement of SHORT-ROOT. Plant Physiol. 176: 392-405.

Tian, J., Han, L., Feng, Z., Wang, G., Liu, W., Ma, Y., Yu, Y. and Kong, Z. 2015. Orchestration of microtubules and the actin cytoskeleton in trichome cell shape determination by a plant-unique kinesin. eLife 4: e09351.

Tseng, K. F., Wang, P., Lee, Y. J., Bowen, J., Gicking, A. M., Guo, L., Liu, B. and Qiu, W. 2018. The preprophase band-associated kinesin-14 OsKCH2 is a processive minus-end-directed microtubule motor. Nat. Commun. 9: 1067.

Umezu, N., Umeki, N., Mitsui, T., Kondo, K. and Maruta, S. 2011. Characterization of a novel rice kinesin $\mathrm{O} 12$ with a calponin homology domain. J. Biochem. 149: 91-101.

Vale, R. D. 2003. The molecular motor toolbox for intracellular transport. Cell 112: 467-480.

Walter, W. J., Machens, I., Rafieian, F. and Diez, S. 2015. The non-processive rice kinesin-14 OsKCH1 transports actin filaments along microtubules with two distinct velocities. Nat. Plants 1: 15111.

Xu, T., Qu, Z., Yang, X., Qin, X., Xiong, J., Wang, Y., Ren, D. and Liu, G. 2009. A cotton kinesin GhKCH2 interacts with both microtubules and microfilaments. Biochem. J. 421: 171-180.

Yamada, M., Tanaka-Takiguchi, Y., Hayashi, M., Nishina, M. and Goshima, G. 2017. Multiple kinesin-14 family members drive microtubule minus end-directed transport in plant cells. J. Cell Biol. 216: 1705-1714.

Yamada, M. and Goshima, G. 2018. The $\mathrm{KCH}$ kinesin drives nuclear transport and cytoskeletal coalescence to promote tip cell growth in Physcomitrella patens. Plant Cell 30: 1496-1510.

Yang, X.-Y., Chen, Z.-W., Xu, T., Qu, Z., Pan, X.-D., Qin, X.-H., Ren, D.-T. and Liu, G.-Q. 2011. Arabidopsis kinesin KP1 specifically interacts with VDAC3, a mitochondrial protein, and regulates respiration during seed germination at low temperature. Plant Cell 23: 1093-1106.

Yasuhara, H. and Kitamoto, K. 2014. Aphidicolin-induced nuclear elongation in tobacco BY-2 cells. Plant Cell Physiol. 55: 913-927.

Yasuhara, H. and Kurisu, W. 2019. A kinesin-related protein, TBK11, associates with the nuclear envelope throughout the cell cycle in tobacco BY-2 cells. Cytologia 84: 277-283.

Yasuhara, H. and Oe, Y. 2011. TMBP200, a XMAP215 homologue of tobacco BY-2 cells, has an essential role in plant mitosis. Protoplasma 248: 493-502.

Yoneda, A. and Hasezawa, S. 2003. Origin of cortical microtubules organized at $\mathrm{M} / \mathrm{G}_{1}$ interface: Recruitment of tubulin from phragmoplast to nascent microtubules. Eur. J. Cell Biol. 82: 461-471. 